\title{
NUTRICIÓN HOSPITALARIA Y AMBULATORIA EN ENFERMOS CON COVID-19.
}

\author{
HOSPITAL AND OUTPATIENT NUTRITION IN PATIENTS WITH COVID-19.
}

Covarrubias Gómez Alfredo ${ }^{1,2}$, Arellano Carreño Víctor Alan ${ }^{3}$, Uscanga Castillo Juan David ${ }^{3}$, Rosas Romero Reina ${ }^{1}$.

1 Instituto Nacional de Ciencias Médicas y Nutrición Salvador Zubirán. 2 Centro Algia para la Educación en Salud. 3 Hospital Henri Dunant, México.

Citation: Covarrubias Gómez A., Arellano Carreño V.A., Uscanga Castillo J.D., Rosas Romero Reina. (2020) Nutrición hospitalaria y ambulatoria en enfermos con COVID-19. Revista Salud Pública y Nutrición, 19 (3), 28-37.

Editor: Esteban G. Ramos Peña, Dr. CS., Universidad Autónoma de Nuevo León, Facultad de Salud Pública y Nutrición, Monterrey Nuevo León, México. Copyright: (C2020 Covarrubias Gómez A., et al. This is an open-access article distributed under the terms of Creative Commons Attribution License [CC BY 4.0], which permits unrestricted use, distribution, and reproduction in any medium, provided the original author and source are credited.

Competing interests: The authors have declared that no competing interests exist.

DOI: https://doi.org/10.29105/respyn19.3-4

Recibido: 03 de junio 2020;

Aceptado: 01 de septiembre 2020

Email: alfredo.covarrubias@algia.org.mx 


\title{
NUTRICIÓN HOSPITALARIA Y AMBULATORIA EN ENFERMOS CON COVID-19.
}

\author{
Covarrubias-Gómez Alfredo ${ }^{1,2}$, Arellano-Carreño Víctor Alan³, Uscanga-Castillo Juan David ${ }^{3}$, Rosas-Romero \\ Reina ${ }^{1}$.
}

1 Instituto Nacional de Ciencias Médicas y Nutrición Salvador Zubirán. 2 Centro Algia para la Educación en Salud. 3 Hospital Henri Dunant, México.

\section{RESUMEN}

Introducción. La enfermedad por SARS-CoV-2 ha cambiado nuestra percepción de la atención hospitalaria e involucra el contexto médico y el nutricional. Las decisiones clínicas respecto al plan nutricional de un enfermo ocurren en un medio multidisciplinario. Objetivo: Proporcionar a los clínicos elementos necesarios para realizar sugerencias nutricionales fundamentadas en la evidencia para enfermos con COVID-19. Material y Método: Se presenta un caso clínico. Se revisa la literatura disponible sobre la nutrición ambulatoria y hospitalaria de enfermos con COVID-19. Se discuten aspectos relacionados con la nutrición en esta población en el contexto hospitalario y ambulatorio. Resultados: Se revisaron diferentes guías internacionales sobre la nutrición en enfermos con COVID-19 en el contexto hospitalario y ambulatorio. Entre las recomendaciones destacan: (i) consideraciones sobre la nutrición enteral temprana y su manejo durante la pronación, (ii) las medidas ambulatorias del enfermo con COVID-19, y (iii) el impacto de la enfermedad en los servicios de alimentos hospitalarios. Conclusiones: Los servicios de alimentación hospitalaria son un área vulnerable durante una pandemia. Los protocolos nutricionales si bien son semejantes a los realizados en unidades críticas deben más orientados a los diferentes momentos que viven los enfermos COVID-19. Se requiere mayor investigación y esta experiencia constituye un área de oportunidad.

Palabras Clave: Nutrición; COVID-19; Hospital; Ambulatorio; Distintivo H.

\section{ABSTRACT}

Introduction: SARS-COV-2 disease has changed our perception of hospital care and it includes the medical and nutritional context. Clinical decision making about patient's nutrition occurs in a multidisciplinary setting. Objective: Provide the basic elements about nutritional evidence-based suggestions on COVID-19 to health care professionals. Material and method: A clinical case is presented. The available literature on outpatient and hospital nutrition of patients with COVID-19 is reviewed. Nutritional aspects of this population are discussed in the hospital and outpatient setting. Results: Different international guidelines on nutrition in patients with COVID-19 in the hospital and outpatient context were reviewed. Among the recommendations are: (i) considerations on early enteral nutrition and its management during pronation, (ii) the outpatient measures of the patient with COVID-19, and (iii) the impact of the disease on hospital food services. Conclusions: Hospital feeding services are a vulnerable area during a pandemic. Although the nutritional protocols are similar to those carried out in critical units, they should be more oriented to the different moments experienced by COVID-19 patients. Further research is required, and this experience constitutes an area of opportunity.

Key words: Nutrition; COVID-19; Hospital; Ambulatory; Distinctive H. 


\section{Introducción}

La enfermedad por SARS-CoV-2 denominada COVID-19 está cambiando la forma de conceptualizar la atención en salud en el mundo y la nutrición hospitalaria o ambulatoria no son excepciones (Lavianno, Koverech, \& Zanetti, 2020) (Butler \& Barrientos, 2020). Esta enfermedad está causada por una familia viral denominada coronavirus. Los coronavirus producen enfermedades de las vías respiratorias con diferentes niveles de severidad (por ejemplo, el Severe Acute Respirtory Syndrome o SARS del 2003) (World Health Organization, 2020).

El COVID-19 recibe ese nombre del acrónimo "Coronavirus Disease 2019" y es causada por el SARS-Cov-2. Esta enfermedad condiciona la presencia de un Síndrome de Distress Respiratorio Agudo (Acute Respiratory Distress Syndrome o ARDS) (World Health Organization, 2020). En el mundo, al 22 de julio del 2020, se habían contagiado cerca de 15.4 millones de personas $(0.2 \%$ de la población mundial) y habían muerto más de 632,000 personas. En México, al 22 de julio del 2020, se habían infectado cerca de 362,000 personas y habían muerto cerca de 41,190 personas (tasa de mortalidad mayor al 11\%).

El hecho de que en poco tiempo se enfermen un gran número de sujetos condiciona que los sistemas de salud puedan colapsarse. Los hospitales no solo pueden saturarse en ocupación sino sobrepasar sus recursos humanos y materiales. Estos incluyen desde las terapias intensivas hasta los servicios de alimentos (Lavianno, Koverech, \& Zanetti, 2020) (Butler \& Barrientos, 2020).

Los hospitales para asegurar la inocuidad de los alimentos que se proporcionan deben prolongar al máximo las fases de latencia y aceleración positiva. Esto se alcanza mediante: (i) lograr que los alimentos contengan microorganismos dentro de los límites permitidos por las normas ya establecidas; (ii) evitar la contaminación alimentaria pon recipientes y utensilios contaminados; (iii) crear condiciones desfavorables para el crecimiento microbiano; y (iv) proporcionar elementos que reduzca el número de microorganismos por acción directa (por ejemplo, la radiación) (Ecured, s. f.) (Gobierno de México, 1995).
En el contexto del COVID-19 la contaminación puede presentarse en los servicios de alimentos por los proveedores de servicios e insumos; el personal que labora en el departamento de servicio de alimentos; el contacto de charolas, vajillas y cubiertos de pacientes infectados; las superficies de preparación; entre muchas otras más. Consecuentemente se deben extremar medidas para salvaguardar la seguridad de los enfermos y del personal.

En cuanto la atención nutricional en el ámbito hospitalario podemos considerar a la evaluación nutricional como "la obtención de información clínica relacionada con la alimentación del paciente". La evaluación consta de: (i) La obtención de datos e información por parte del individuo evaluado. (ii) $\mathrm{La}$ realización de pruebas y mediciones. (iii) Aplicación sistemática y ordenada de los datos. (iv) Evaluación e interpretación de los datos, pruebas obtenidas. (v) Diagnóstico nutricional.

El objetivo del presente trabajo es proporcionar a los clínicos información fundamentada en la evidencia acerca de aspectos nutricionales relacionados específicamente a enfermos con infección causada por el SARS-Cov-2 para facilitar su toma de decisiones en el futuro.

\section{Caso clínico en nutrición}

Se trata de una mujer de 43 años originaria de la Ciudad de México. Cuenta con los diagnósticos previos de hipertensión arterial de 10 años de evolución en tratamiento y control con candesartán y diabetes mellitus 2 de 7 años de evolución en tratamiento y pobre control con metformina. Es sedentaria. Niega alcoholismo, tabaquismo y otras toxicomanías. No presenta otros antecedentes de relevancia.

Acude al servicio de urgencias por dificultad respiratoria, sensación de ahogo, y fiebre de 39 grados centígrados que iniciaron 24 horas antes de su llegada al hospital.

Cuenta con un peso de 100 kilogramos, una estatura de 165 centímetros, frecuencia cardiaca de 100 latidos por minuto, frecuencia respiratoria de 45 respiraciones por minuto, una tensión arterial de 170/100 milímetros de mercurio, temperatura de 39 grados centígrados, y $\mathrm{SpO} 2$ de $79 \%$. Presenta 
Glasgow de 15 y se encuentra orientada en tiempo, persona y lugar.

Se encontró con franca dificultad respiratoria (taquipnea, tiraje intercostal, actividad de músculos accesorios) y síndrome febril (temperatura corporal de 39 grados centígrados, taquicardia de 100 latidos por minuto, diaforesis). A la exploración se encuentra palidez de mucosas con buena hidratación, eritema conjuntival, epífora, taquicardia sin algún otro fenómeno a la auscultación, taquipnea con bases congestivas e hipoventiladas en forma bilateral, cianosis peribucal y acrocianosis distal, el resto de la exploración se encuentra dentro de parámetros normales.

Se le realizó una tomografía axial computada en la que se documenta neumonía compatible con enfermedad por SARS-CoV-2. La reacción en cadena de la polimerasa con transcriptasa inversa (RT-PCR en inglés) fue positiva a SARS-CoV-2 estableciéndose el diagnóstico de COVID-19.

La evaluación nutricional documenta que la enferma presenta un IMC de $36.73 \mathrm{gr} / \mathrm{cm}$; con ello, se integran los diagnósticos de obesidad grado 2, diabetes mellitus 2, hipertensión arterial, y posible síndrome metabólico. Cabe destacar que ambas situaciones clínicas son considerados factores de riesgo para la enfermedad causada por el SARS COV-2.

Entre los elementos bioquímicos presenta una hemoglobina glicosilada de 10, la función renal y hepática se encuentran conservadas, y no cuenta con elementos gasométricos que indiquen intubación. Tiene un valor de proteína $C$ reactiva de 10 .

Desde el punto de vista de la evaluación dietética no existe pérdida de peso o ni del apetito en los últimos 3 meses. Las alergias e intolerancias alimenticias son negativas. Se identifican diversos riesgos: (i) riesgo de desnutrición por neumonía atípica, (ii) riesgo inflamatorio por obesidad, y (iii) riesgo metabólico por diabetes mellitus 2. Estas consideraciones sugieren que la enferma requiere de un seguimiento nutricional post-hospitalización.

Se proporcionó manejo con mascarilla de alto flujo con 15 litros de oxígeno por minuto (con saturación de $88 \%$ y gasometría arterial sin criterios de intubación), no toleró la pronación, se proporcionan antiretrovirales (remdesivir e hidroxicloroquina) y antiagregación plaquetaria (ácido acetil-salicílico). La enferma después de 10 días evoluciona hacia la mejoría y es egresada del hospital a los 17 días posterior a su ingreso. Se programa valoración subsecuente en consulta externa post-COVID.

\section{Consideraciones nutricionales}

Generalidades.

A mediados de diciembre de 2019, en la ciudad de Wuhan, en el centro de China, se declaró una epidemia de coronavirus. Esta enfermedad es causada por un nuevo tipo de coronavirus llamado primero 2019-nCoV, luego renombrado SARS-CoV2 , nunca observado antes de su aparición en esta metrópoli de 11 millones de habitantes (Saqrane \& El Mhammedi, 2020).

De tal forma que la enfermedad de COVID-19 es causada por el SARS-CoV-2. Este es un virus que pertenece al gran coronavirus. Los coronavirus pueden causar desde un resfriado simple hasta una infección respiratoria grave como la neumonía. Esta familia vírica, ha causado epidemias fatales, tal fue el caso del SRAS o MERS y ahora del COVID-19 (Saqrane \& El Mhammedi, 2020).

Como cualquier pandemia, esta enfermedad tiene distribución global. Tiene una tasa de mortalidad entre 3 y $10 \%$. Los países europeos han sido severamente afectados, principalmente Italia y España (Saqrane \& El Mhammedi, 2020). En el Reino Unido, por ejemplo, se ha identificado que afecta más a los hombres, con mayor Índice de Masa Corporal, la mayoría de los enfermos era capaz de autocuidarse, la cantidad de intubaciones se relaciona con obesidad y otras comorbilidades (principalmente afección pulmonar crónica, cardiovascular y diabetes), los enfermos con intubación la mantienen de 5 a 14 días (Phua, y otros, 2020).

Conforme a lo que se ha repostado de la experiencia hospitalaria de enfermos con COVID-19, es posible identificar los siguientes grupos: (i) los enfermos que tienen con enfermedad moderada y no requieren pronación ni intubación; (ii) los que tienen con enfermedad severa y requieren pronación, pero no intubación; (iii) los que tienen enfermedad severa y requieren pronación e intubación. (Phua, y otros, 2020) (Saqrane \& El Mhammedi, 2020). 
Con base en cada uno de estos grupos se han propuesto diferentes esquemas de ministración de los alimentos entre los que se encuentran la vía oral, la enteral, y la parenteral. Cada uno de estos abordajes ocurre en diferentes sitios al interior del hospital y requerirán la participación del personal de servicios de alimentos y de los nutriólogos clínicos (Tabla 1) (ASPEN-SCCM, 2020) (Lavianno, Koverech, \& Zanetti, 2020) (Barazzoni, y otros, 2020). Nunca debemos olvidar que todos producimos aerosoles y que los aerosoles son los transmisores del virus (Herron, Hay-David, Gilliam, \& Brennan, 2020). Todos tienen COVID-19 hasta demostrar lo contrario.

Tabla 1. Consideraciones nutricionales en los diferentes grupos de enfermos con COVID-19 en el contexto hospitalario

Enfermedad moderada y no requieren pronación ni intubación.

Sitio en hospital: Piso de hospitalización o terapia intermedia Nutrición oral (hipercalórica e hiperprotéica)

Nutrición enteral

Enfermedad severa y requieren pronación pero no intubación.

Sitio en hospital: Terapia intermedia o terapia intensiva Nutrición enteral temprana

Enfermedad severa y requieren pronación e intubación. Sitio en hospital: Terapia intensiva

Nutrición enteral temprana

Nutrición parenteral (fórmulas poliméricas)

Realizada por el Dr. Alfredo Covarrubias-Gómez con información de (Lavianno, Koverech, \& Zanetti, 2020).

Recomendaciones nutricionales en sujetos con enfermedad severa que se encuentran en la terapia intermedia o intensiva.

Es mandatorio que antes de cualquier intervención en enfermos con diagnóstico de COVID-19 se sigan los estándares de colocación de los EPP [equipo de protección personal] establecidos por los CDC de los EE. UU. [Centers for Disease Control]. Esta regla es aplicable para todos los proveedores de atención en salud, incluidos los médicos, nutriólogos, enfermeras y demás profesionales de la salud. El EPP incluye: (i) gafas protectoras o "googles", (ii) bata de aislamiento u "overol", (iii) un protector facial o "careta facial", y (iv) un respirador N95 (Figura 1) (ASPEN-SCCM, 2020) (Barazzoni, y otros, 2020) (Phua, y otros, 2020) (Lavianno, Koverech, \& Zanetti, 2020) (Herron, Hay-David, Gilliam, \& Brennan, 2020).
Figura 1. Equipo de protección personal (EPP) para los profesionales de la salud.

\section{PROTECCION DEL PERSONAL}

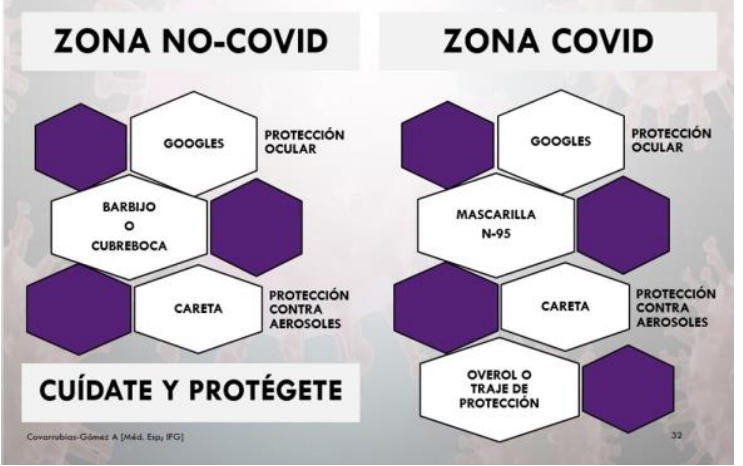

La imagen muestra una infografía para ser colocada en las áreas COVID y No-COVID a nivel hospitalaria enfocada a los trabajadores de la salud por el Dr. Alfredo Covarrubias-Gómez con información de (Lavianno, Koverech, \& Zanetti, 2020).

Todos los grupos coinciden en que la evaluación nutricional y el tamizaje del riesgo nutricional es importante en los enfermos con COVID-19. Esta evaluación $\mathrm{ABCD}$ debe además contemplar todas las condiciones comórbidas, la función del tracto gastrointestinal y el riesgo de aspiración (ABCD es una nemotécnica para la evaluación nutricional que hace referencia a parámetros: Antropométricos, Bioquímicos, Clínicos, Dietéticos). Se ha sugerido que no se utilicen indicadores de nutrición tradicionales o marcadores sustitutos, ya que no están validados en cuidados críticos. La evaluación del riesgo nutricional se puede realizar con dos instrumentos validados como el Nutritional Risk Screening [NRS 2002] (utilizado en el ámbito del enfermo geriátrico, considera la severidad de la enfermedad y un estado nutricional de riesgo) (Tabla 2) y el NUTRIC score (utilizado comúnmente en la terapia intensiva) (Tabla 3) (ASPEN-SCCM, 2020) (Barazzoni, y otros, 2020) (Phua, y otros, 2020) (Lavianno, Koverech, \& Zanetti, 2020) (Herron, Hay-David, Gilliam, \& Brennan, 2020). 


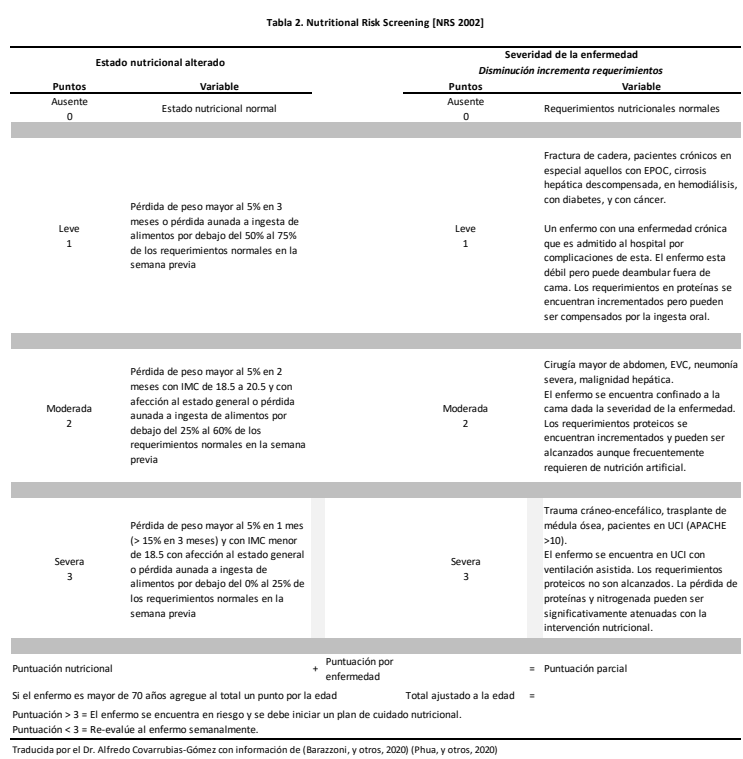

Tabla 3. Variables evaluadas por el NUTRIC score.*

\begin{tabular}{|c|c|c|}
\hline Variable & Rango & Puntos \\
\hline \multirow{3}{*}{ Edad } & $<50$ & 0 \\
\hline & $50 a<75$ & 1 \\
\hline & $>75$ & 2 \\
\hline \multirow{4}{*}{ APACHE II } & $<15$ & 0 \\
\hline & $15 a<20$ & 1 \\
\hline & 20 a 28 & 2 \\
\hline & $>28$ & 3 \\
\hline \multirow{3}{*}{ SOFA } & $<6$ & 0 \\
\hline & $6 a<10$ & 1 \\
\hline & $>10$ & 2 \\
\hline \multirow{2}{*}{$\begin{array}{l}\text { Número de } \\
\text { comorbilidades }\end{array}$} & 0 a 1 & 0 \\
\hline & 20 más & 1 \\
\hline \multirow{2}{*}{$\begin{array}{c}\text { Días de estancia } \\
\text { en la UCl }\end{array}$} & 0 a 1 & 0 \\
\hline & 20 más & 1 \\
\hline \multirow{2}{*}{ IL-6 } & $0 \mathrm{a}<400$ & 0 \\
\hline & $>400$ & 1 \\
\hline
\end{tabular}

Continúa... Tabla 3. Variables evaluadas por el NUTRIC score. ${ }^{*}$
Continuación... Tabla 3. Variables evaluadas por el NUTRIC score.

\begin{tabular}{|c|c|c|c|}
\hline \multicolumn{4}{|c|}{ Sistema de puntuación su la IL-6 está disponible } \\
\hline Suma de puntos & Categoría & Explicación & Explicación \\
\hline 6а 10 & Puntuación alta & $\begin{array}{l}\text { Asociada con un mal } \\
\text { desenlace clínico } \\
\text { (mortalidad, ventilación). } \\
\text { Estos enfermos se } \\
\text { benefician con una terapia } \\
\text { nutricional agresiva }\end{array}$ & $\begin{array}{l}\text { Asociada con un mal } \\
\text { desenlace clínico } \\
\text { (mortalidad, } \\
\text { ventilación). } \\
\text { Estos enfermos se } \\
\text { benefician con una } \\
\text { terapia nutricional } \\
\text { agresiva }\end{array}$ \\
\hline 0 a 5 & Puntuación baja & $\begin{array}{l}\text { Estos enfermos tienen un } \\
\text { riesgo bajo de } \\
\text { malnutrición }\end{array}$ & $\begin{array}{l}\text { Estos enfermos } \\
\text { tienen un riesgo bajo } \\
\text { de malnutrición }\end{array}$ \\
\hline \multicolumn{4}{|c|}{ Sistema de puntuación su la IL-6 NO está disponible** } \\
\hline Suma de puntos & Categoría & Explicación & Explicación \\
\hline 5 a 9 & Puntuación alta & $\begin{array}{l}\text { Asociada con un mal } \\
\text { desenlace clínico } \\
\text { (mortalidad, ventilación). } \\
\text { Estos enfermos se } \\
\text { benefician con una terapia } \\
\text { nutricional agresiva }\end{array}$ & $\begin{array}{l}\text { Asociada con un mal } \\
\text { desenlace clínico } \\
\text { (mortalidad, } \\
\text { ventilación). } \\
\text { Estos enfermos se } \\
\text { benefician con una } \\
\text { terapia nutricional } \\
\text { agresiva }\end{array}$ \\
\hline 0 a 4 & Puntuación baja & $\begin{array}{l}\text { Estos enfermos tienen un } \\
\text { riesgo bajo de } \\
\text { malnutrición }\end{array}$ & $\begin{array}{l}\text { Estos enfermos } \\
\text { tienen un riesgo bajo } \\
\text { de malnutrición }\end{array}$ \\
\hline
\end{tabular}

Traducida por el Dr. Alfredo Covarrubias-Gómez con información de (Barazzoni, y otros, 2020) (Phua, y otros, 2020)

* El NUTRIC Score está diseñado para realizar el tamizaje del riesgo nutricional en pacientes críticamente enfermos y que desarrollan eventos que pueden ser modificados con una terapia nutricional agresiva. La puntuación va de 0 a 10 puntos. ** Si bien la IL-6 es un indicador de severidad en el enfermo críticamente enfermo su papel en el NUTRIC Score es mínimo y no es esencial solicitarla.

Con respecto al cálculo de los requerimientos energéticos de los enfermos con COVID-19, algunos autores sugieren que puede emplearse la calorimetría indirecta en los centros en donde se cuente con ella y que además sea operativamente factible su realización (ASPEN-SCCM, 2020) (Barazzoni, y otros, 2020) (Phua, y otros, 2020) (Lavianno, Koverech, \& Zanetti, 2020) (Herron, Hay-David, Gilliam, \& Brennan, 2020) [se debe considerar que es muy alta la posibilidad de que el virus se aloje en la cámara del calorímetro; las mangueras y cámaras utilizadas en estos equipos son desechables en los EE. UU. y estos insumos deben ser tratados como material con riesgo biológico letal]. Si esto no es posible, puede considerarse un cálculo de 25 $\mathrm{kcal} / \mathrm{kg} / \mathrm{día}$ el cual puede ser empleado durante cinco días, en aquellos pacientes con más de 5 a 7 días hospitalización el cálculo puede ser realizado considerando $30 \mathrm{kcal} / \mathrm{kg} / \mathrm{día}$ (ASPEN-SCCM, 2020) (Barazzoni, y otros, 2020) (Phua, y otros, 2020) (Lavianno, Koverech, \& Zanetti, 2020) (Herron, Hay-David, Gilliam, \& Brennan, 2020). 
El aporte calórico debe considerar también la administración de fármacos o soluciones (por ejemplo; proporfol, soluciones mixtas, soluciones de hidroxietil-almidón, entre otras). Así mismo, el cálculo proteico en estos enfermos debe considerar de 1.2 a $2.0 \mathrm{~g} / \mathrm{kg} / \mathrm{día}$ (ASPEN-SCCM, 2020) (Barazzoni, y otros, 2020) (Phua, y otros, 2020) (Lavianno, Koverech, \& Zanetti, 2020) (Herron, Hay-David, Gilliam, \& Brennan, 2020).

El paciente hospitalizado en las unidades de cuidados intensivos (UCI) con COVID-19 debe iniciar una terapia de soporte nutricional en forma temprana, en especial en aquellos que no pueden mantener ingesta volitiva, la nutrición enteral debe ser iniciada en un plazo de 24 a 48 horas posterior al ingreso a la UCI (ASPEN-SCCM, 2020) (Barazzoni, y otros, 2020) (Phua, y otros, 2020) (Lavianno, Koverech, \& Zanetti, 2020) (Herron, Hay-David, Gilliam, \& Brennan, 2020).

El inicio de la nutrición enteral debe iniciarse en todos los enfermos con COVID-19 tempranamente (Figura 2).

Figura 2. Manejo nutricional en individuos hospitalizados con riesgo de COVID-19, aquellos que ya cuentan con la enfermedad, y aquellos ingresados en la UCI.

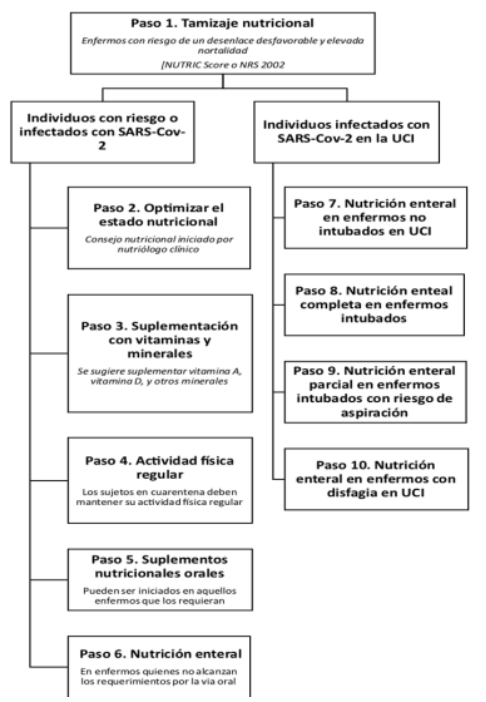

La imagen muestra la secuencia de pasos a implementarse en enfermos con COVID-19 en distintos contextos.
No se considera la presencia de peristaltismo para su inicio y se prefiere la nutrición enteral sobre la parenteral. Es destacable el hecho de que del $30 \%$ al $70 \%$ de los pacientes ingresados en la UCI presentarán disfunción gastrointestinal secundaria; este fenómeno clínico depende del diagnóstico establecido, las condiciones pre-mórbidas, el modo de ventilación, los medicamentos ministrados y el estado metabólico (ASPEN-SCCM, 2020) (Barazzoni, y otros, 2020) (Phua, y otros, 2020) (Lavianno, Koverech, \& Zanetti, 2020) (Herron, Hay-David, Gilliam, \& Brennan, 2020).

Al iniciar la nutrición enteral debemos considerar que el nivel de infusión se desvía más bajo en el tracto gastrointestinal. En los enfermos críticos con alto riesgo de aspiración o en aquellos que han mostrado intolerancia a la nutrición enteral gástrica se debe poner especial atención al volumen ministrado (Tabla 4).

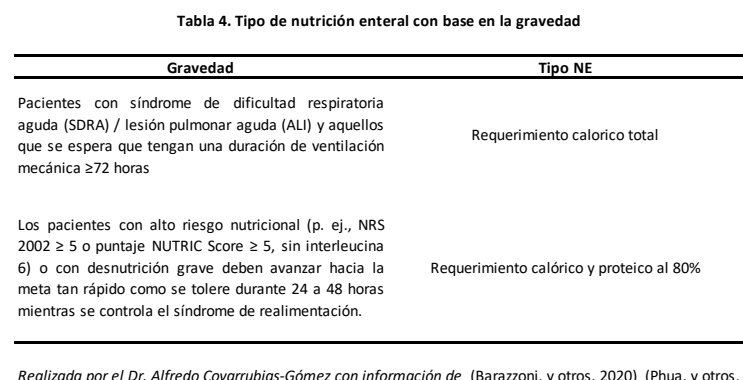
2020)

Este no debe exceder los 300 a $500 \mathrm{ml} /$ día con una densidad energética aproximada de 1.25 a 1.5 $\mathrm{kcal} / \mathrm{ml}$. La alimentación enteral trófica (generalmente definidas como $10-20 \mathrm{ml} / \mathrm{hr}$ o $10-20$ $\mathrm{kcal} / \mathrm{hr}$ ) puede ser suficiente para prevenir la atrofia de la mucosa y mantener la integridad intestinal [existe la posibilidad de que los requerimientos nutricionales no sean alcanzados con esa estrategia y podría sugerirse la implementación de una suplementación parenteral; sin embargo, esta recomendación no es considerada en las guías consultadas para la realización de esta revisión] (ASPEN-SCCM, 2020) (Barazzoni, y otros, 2020) (Phua, y otros, 2020) (Lavianno, Koverech, \& Zanetti, 2020) (Herron, Hay-David, Gilliam, \& Brennan, 2020).

Dada la heterogeneidad de los protocolos para la nutrición enteral en el sujeto pronado y críticamente 
enfermo, la factibilidad de realizar recomendaciones categóricas es limitada. En el contexto específico del enfermo pronado con COVID-19 las recomendaciones conjuntas de la ASPEN-SCCM sugieren suspender la nutrición enteral en aquellos enfermos que presentan compromiso o inestabilidad hemodinámica (estado de choque) y reiniciarla hasta que el enfermo se estabilice. La disminución del flujo sanguíneo intestinal por hipoperfusión puede condicionar isquemia y necrosis contraindicando la nutrición enteral. Consecuentemente es importante tener precaución en enfermos que presentaron hipoperfusión y que reciben vasopresores (ASPENSCCM, 2020) (Barazzoni, y otros, 2020) (Phua, y otros, 2020) (Lavianno, Koverech, \& Zanetti, 2020) (Herron, Hay-David, Gilliam, \& Brennan, 2020).

En los enfermos en pronación también se debe suspender la nutrición enteral durante el tiempo que dure pronado, cuando esta termina se debe corroborar la posición del tubo de nutrición. En ellos, también se debe considerar el uso de procinéticos por el riesgo de aspiración (metoclopramida) y al reiniciar la nutrición procurar mantener la cabeza de $30^{\circ}$ a $45^{\circ}$. En los pacientes con puntas nasales de alto flujo se debe considerar que la irritación de la mucosa puede condicionar náusea y distensión gástrica (ASPEN-SCCM, 2020) (Barazzoni, y otros, 2020) (Phua, y otros, 2020) (Lavianno, Koverech, \& Zanetti, 2020) (Herron, Hay-David, Gilliam, \& Brennan, 2020).

Con respecto a los líquidos es importante mencionar que no deben restringirse; sin embargo, se debe vigilar la ingesta hídrica y el aporte de líquidos por otras vías (líquidos o nutrición parenterales, nutrición enteral). La presencia de sobrecarga hídrica y edema pulmonar puede empeorar la neumonitis severa del enfermo con COVID-19 e incrementar la disnea. Del mismo modo, se sugiere que las formulaciones enterales contengan poco líquido ya que retrasan el vaciamiento gástrico y conducen a intolerancia. En el paciente geriátrico se debe vigilar el sodio por el riesgo de hiponatremia y en enfermo con nutrición enteral existe riesgo de hipernatremia (ASPEN-SCCM, 2020) (Barazzoni, y otros, 2020) (Phua, y otros, 2020) (Lavianno, Koverech, \& Zanetti, 2020) (Herron, Hay-David, Gilliam, \& Brennan, 2020). Ninguna de las recomendaciones sobre nutrición en el enfermo con COVID-19 realizada por la ASPEN-SCCM, ESPEN, y AusPEN recomienda alguna formulación enteral específica al momento.

Recomendaciones nutricionales en sujetos con enfermedad leve que se encuentran en hospitalización (área no-intensiva) o en domicilio. En los enfermos que se encuentran en domicilio o que se encuentran en hospitalización y que no tienen criterios de gravedad ni requieren ventilación invasiva o pronación, la ingesta oral es fundamental. El objetivo es satisfacer las necesidades nutricionales considerando que están cursando con una enfermedad infecciosa que promueve un estado hipermetabólico (ASPEN-SCCM, 2020) (Phua, y otros, 2020).

En ese sentido el gasto energético basal puede estimarse entre 2,000 y 2,500 calorías por día. El estado hipermetabólico y las modificaciones en el gusto y el olfato pueden condicionar que los enfermos con COVID-19 pierdan el apetito. Se ha sugerido que el régimen alimenticio este dividido en sextos. Es conveniente recordar que las calorías son importantes para proteger al músculo contra la degradación proteica para obtener energía; esto aunado al estrés metabólico causado por el COVID19 , permite una ingesta calórica mayor a la contenida en la dieta habitual (ASPEN-SCCM, 2020).

El consumo de proteína debe ser elevado por lo que se estima entre 75 y 100 gramos de proteína por día. El consumo de líquidos debe ser de alrededor de 3 litros de líquido por día. Los líquidos óptimos para beber son las bebidas líquidas claras con calorías y proteínas, soluciones de rehidratación oral o bebidas deportivas con proteína añadida. Se recomienda el consumo de 60 a 120 mililitros cada 15 a 20 minutos (ASPEN-SCCM, 2020) (Figura 3). 
Figura 3. Infografía sugerida para el enfermo con COVID-19 en domicilio o en piso de hospitalización y que no tienen criterios de gravedad ni requieren ventilación invasiva o pronación.

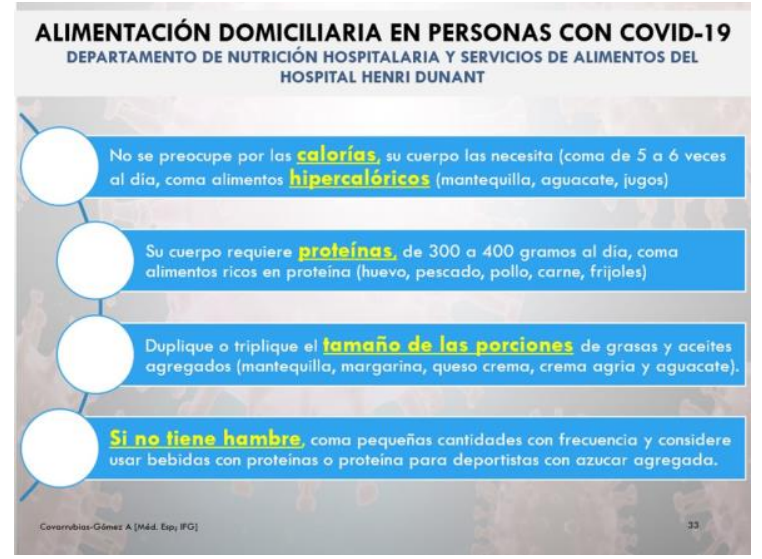

La imagen muestra una infografía que pueda ser proporcionada a los enfermos con COVID-19 en domicilio o en piso de hospitalización y que no tienen criterios de gravedad ni requieren ventilación invasiva o pronación. Esta serie de recomendaciones solo aplican 10 a 14 días de enfermedad confirmada. No aplica en enfermos con diabetes, sobrepeso, hipertensión, nefropatías o hepatopatías. Realizada por el Dr. Alfredo Covarrubias-Gómez con información de (Lavianno, Koverech, \& Zanetti, 2020).

Evolución del caso expuesto.

Atendiendo a las recomendaciones propuestas por la ASPEN-SCCM y ESPEN encontramos dos escenarios. El primero es la nutrición al internamiento y el segundo es la nutrición en domicilio.

Sobre la alimentación durante el internamiento debemos recordar que el caso propone a una enferma con COVID-19 con diabetes mellitus tipo 2, hipertensión, obesidad grado 2, probable síndrome metabólico y COVID-19. Esta persona pese a una saturación deficiente $(\mathrm{SpO} 2$ del $88 \%)$ no toleró la pronación y se mantuvo con puntas nasales de alto flujo como apoyo ventilatorio no invasivo. El apetito de la enferma se encuentra disminuido en un $50 \%$ debido a los equipos no invasivos de ventilación, al malestar generalizado de la enferma, y a las alteraciones del gusto y del olfato asociados a la enfermedad. También es importante destacar que la enferma requirió de cuidados intermedios y no de cuidados intensivos.

En el paciente hospitalizado con COVID-19 el requerimiento energético toma en consideración un cálculo de 25 a $30 \mathrm{kcal} / \mathrm{kg} / \mathrm{día}$; si nuestra enferma pesa $100 \mathrm{~kg}$ con una talla de $165 \mathrm{~cm}$ podemos considerar su peso ideal utilizando los rangos de un índice de masa corporal saludable (por este medio peso se encuentra entre 50.4 y 68.1 kilogramos [para un IMC de 25 el peso ideal sería de 68 kilos]).

Con lo anterior podemos estimar un requerimiento energético basal de $1,700 \mathrm{kcal} /$ día del día 1 del inicio de la enfermedad al día 7 y de 2,040 kcal/día del día 8 al día 15 del inicio de la enfermedad (ASPENSCCM, 2020) (Barazzoni, y otros, 2020) (Phua, y otros, 2020) (Lavianno, Koverech, \& Zanetti, 2020) (Herron, Hay-David, Gilliam, \& Brennan, 2020).

Después del día 15 al 21 del inicio de la enfermedad el requerimiento energético basal vuelve a las condiciones previas al evento y en el caso de que no presente alguna complicación médica (en el caso de la enferma podemos contemplar una dieta modificada en macro- y micro-nutrientes dados sus diagnósticos nutricionales) (ASPEN-SCCM, 2020) (Barazzoni, y otros, 2020) (Phua, y otros, 2020) (Lavianno, Koverech, \& Zanetti, 2020) (Herron, Hay-David, Gilliam, \& Brennan, 2020). Diversos autores sugieren que el peso contemplado en el cálculo debe ser el peso ideal (Thibault, y otros, 2020). En este caso, el seguimiento post-hospitalario no fue posible debido a la gravedad de la enfermedad y la prioridad de atención hospitalaria frente a la contingencia del COVID-19.

La distribución dieto sintética de carbohidratos y lípidos no son consideradas en las recomendaciones realizadas por ASPEN-SCCM y ESPEN; sin embargo, las proteínas contemplan de 1.2 a 2.0 $\mathrm{g} / \mathrm{kg} / \mathrm{d}$ á y se sugiere incrementar los requerimientos de omega-3 (Thibault, y otros, 2020) (Tabla 4). En la enferma del caso los requerimientos proteicos estarían entre de 81.6 a 136 g/día si consideramos su peso para in IMC de 25 .

Las recomendaciones domiciliarias relacionadas con la alimentación de esta enferma no son diferentes a las hospitalarias, poniendo especial énfasis en el consumo de líquidos. 
Aspectos que son importantes considerar son las interacciones alimento medicamento y entre medicamentos. El remdesivir presenta interacciones con el alcohol que pueden condicionar daño hepático. La combinación de remdesivir e hidroxicloroquina presenta interacciones relacionadas con una disminución en la eficacia de remdesivir.

Las alteraciones sobre el olfato y el gusto impactan necesariamente en la percepción de los alimentos y condicionan una disminución en la ingesta. Lo anterior aunado a la pérdida incrementada de peso por el estado hipermetabólico condicionan un riesgo nutricional importante.

Las complicaciones cardiacas, neurológicas, dermatológicas, de coagulación, gastrointestinales, entre otras pueden condicionar eventualidades aún por estudiarse en el corto, mediano y largo plazo. La repercusión nutricional de tales eventualidades aún no ha sido considerada puntualmente.

\section{Conclusiones}

En México pocos hospitales cuentan con Distintivo $\mathrm{H}$. Este reconocimiento es otorgado por la Secretaría de Turismo y es avalado por la Secretaría de Salud. Se entrega a los prestadores de servicios de alimentos y bebidas que cumplen con los estándares definidos por dicho programa. Esto quiere decir que los establecimientos que lo ostentan deben estar apegados a la lista de verificación de la norma vigente (Gobierno de México, 2016).

Si bien es cierto que el Distintivo H garantiza la inocuidad alimentaria, al no tratarse de una norma obligatoria, no es costumbre que los hospitales ostenten tal reconocimiento. Entre los Institutos Nacionales de Salud que cuentan con ese distintivo, se encuentran los siguientes: (i) Psiquiatría; (ii) Rehabilitación; (iii) Neurología y Neurocirugía; (iv) Perinatología; (v) Medicina Genómica; (vi) Cancerología. Cabe destacar que los comedores estos centros se caracterizan por que son provistos por terceros (Gobierno de México, 2016).

Los hospitales que cuentan con el Distintivo H, son capaces de garantizar la inocuidad de los alimentos que son servidos a pacientes, comensales y personal. En esta época de pandemia derivada de la infección por SARS-Cov-2 los protocolos para asegurar la inocuidad requieren ser revisados profundamente. Los hospitales públicos nacionales no tienen la obligación de contar con este distintivo y la única norma que deberían seguir en forma obligatoria es la NOM-251-SSA1-2009 que regula la preparación de los alimentos. El Distintivo $\mathrm{H}$ más allá de ser un reconocimiento de nivel turístico nos ofrece parámetros de calidad más elevados que de la NOM251-SSA1-2009.

Dada la elevada posibilidad de contagio del SARSCov-2 hacia el personal y hacia los pacientes en modelos hospitalarios híbridos, sugerimos que no se pierda de vista una eventual Enfermedad Transmitida por Alimentos a consecuencia de este virus. La situación de pandemia que viven los hospitales nacionales genera la oportunidad de consolidar estrategias tendientes a mejorar la atención de los enfermos sin poner en riesgo al personal.

Entre ellas se encuentra la necesidad de crear modelos educativos acerca del impacto nutricional intrínseco y extrínseco del COVID-19 hacia el personal que labora al interior y exterior de los servicios de alimentación de hospitales híbridos o reconvertidos. En este escenario los nutriólogos clínicos y de servicios de alimentos tienen la oportunidad de generar líneas de investigación en una enfermedad inexplorada y que presenta retos aún desconocidos.

Si bien es cierto que los modelos atención nutricional en una terapia intensiva pueden ser útiles; debemos reconocer que los cambios fisiopatológicos a nivel respiratorio, cardiovascular, neurológico, dermatológico, gastrointestinal y nefrológico (por mencionar algunos) son al momento diferentes a otros procesos de enfermedad y desconocemos el impacto sobre la nutrición humana. Si bien encontramos muchas limitaciones en nuestra búsqueda bibliográfica, también observamos muchas áreas de oportunidad para el desarrollo de la investigación en el campo de la nutriología clínica y de los servicios de alimentación.

\section{Agradecimientos}

Agradecemos a nuestras familias su apoyo incondicional; nuestros pacientes y maestros por su enseñanza; al Centro Algia para la Educación en Salud por las facilidades con la redacción del 
presente trabajo; y al personal del Hospital Henri Dunant por su entrega amorosa a los pacientes y el apoyo proporcionado en esta pandemia.

\section{Bibliografía}

ASPEN-SCCM. (30 de marzo de 2020). Nutrition Therapy in the Patient with COVID-19 Disease Requiring ICU Care. (ASPEN-SCCM) Recuperado el 25 de abril de 2020, de https://www.sccm.org/getattachment/Disaster/N utrition-Therapy-COVID-19-SCCM-

ASPEN.pdf?lang=en-US

Barazzoni, R., Bischoff, S. C., Breda, J., Wickramasinghe, K., Krznaric, Z., Nitzan, D., . . . Singer, P. (2020). ESPEN expert statements and practical guidance for nutritional management of individuals with SARS-CoV-2 infection. Clinical Nutrition (En prensa).

Butler, M. J., \& Barrientos, R. M. (2020). The impact of nutrition on COVID-19 susceptibility and long-term consequences. Brain, behavior, and immunity, En prensa.

Ecured. (s. f.). Conservación de los alimentos. Recuperado el 19 de febrero de 2020, de Ecured: https://www.ecured.cu/Conservaci\%C3\%B3n_d e_alimentos

Gobierno de México. (4 de octubre de 1995). Norma Oficial Mexicana Nom-093-SSA1-1994, Bienes y Servicios. Prácticas de higiene y sanidad en la preparación de alimentos que se ofrecen en establecimientos fijos. Diario Oficial de la Federación.

Gobierno de México. (2016). Gobierno de México. Recuperado el 27 de febrero de 2020, de NMXF-605-NORMEX-2004: Alimentos Manejo Higiénico en el Servicio de Alimentos Preparados para la Obtención del Distintivo H: https://www.gob.mx/cms/uploads/attachment/fil e/197511/NMX-F-605-NORMEX2016_7_de_diciembre_de_2015_firmada_002 -.pdf

Herron, J., Hay-David, A., Gilliam, A. D., \& Brennan, P. A. (2020). Personal protective equipment and COVID-19: A risk to healthcare staff? The British journal of oral \& maxillofacial surgery, S0266-4356(20), 30165-0.

Lavianno, A., Koverech, A., \& Zanetti, M. (2020). Nutrition support in the time of SARS-CoV-2 (COVID-19). Nutrition, En prensa.

Phua, J., Weng, L., Ling, L., Egi, M., Lim, C. M., Divatia, J. V., . . . Du, B. (2020). Intensive care management of coronavirus disease 2019 (COVID-19): challenges and recommendations. Lancet Respiratory Medicine, 8 (5), 506-517.

Saqrane, S., \& El Mhammedi, M. A. (2020). Review on the global epidemiological situation and the efficacy of chloroquine and hydroxychloroquine for the treatment of COVID-19. New microbes and new infections, 35, 100680.

Thibault R, Seguin P, Tamion F, Pichard C, Singer P. Nutrition of the COVID-19 patient in the intensive care unit (ICU): a practical guidance. Crit Care. 2020;24(1):447. Published 2020 Jul 19. doi:10.1186/s13054-020-03159-z

UnADM. (2019). Administración en salud, unidad 2: El proceso administrativo. Ciudad de México, México: UnADM.

UnADM. (2020). Higiene y calidad alimentaria. Unidad 3: Normatividad vigente higiene $y$ calidad alimentaria. Ciudad de México, México: UnADM.

World Health Organization. (2020). SARS (Severe Acute Respiratory Syndrome). Recuperado el 25 de abril de 2020, de https://www.who.int/ith/diseases/sars/en/ 